\title{
Ciencia, Tecnología y Mercado: Investigaciones en Arroz en el INTA Argentino
}

\author{
Cecilia Gárgano ${ }^{1}$
}

Resumen: La importancia del conocimiento en las sociedades contemporáneas, y de su creciente rol en diversas dinámicas productivas y sociales, constituye una temática ampliamente abordada. Asimismo, la disputa por el acceso a distintos bienes comunes naturales es una preocupación de agenda, en particular para las sociedades latinoamericanas. ¿Qué ocurre con la producción de conocimiento científico implicado en la obtención de un recurso crecientemente disputado como las semillas? Este artículo analiza una trayectoria de investigación orientada a la obtención de variedades de arroz resistentes a herbicidas, radicada en el Instituto Nacional de Tecnología Agropecuaria (INTA) argentino. Se exponen las dinámicas de producción y uso de conocimientos científicos y tecnológicos generados con fondos públicos. Mediante documentos institucionales, marcos regulatorios y entrevistas a científicos y técnicos, se analizan las implicancias sociales, económicas y políticas derivadas de la organización de la producción y apropiación del conocimiento, en un sector históricamente relevante como el agro.

Palabras clave: semillas; ciencia; arroz; producción; apropiación; conocimientos científico-tecnológicos; bienes comunes

Abstract: The importance of knowledge in contemporary societies and its growing part in variant productive and social dynamics constitute a widely discussed subject. Additionally, the dispute over the access to various natural common goods is a concern in today's agenda, particularly for Latin American societies. What about the production of scientific knowledge involved in procuring increasingly contended resources such as seeds? This paper studies the trajectory of an investigation into the process of obtaining varieties of rice which are resistant to herbicides, led by the National Institute of Agricultural Technology (INTA), in Argentina. An insight is provided into the dynamics of production and the use of scientific and technological knowledge funded by the government. Based on institutional documentation, regulatory frameworks and a series of interviews with scientists and technicians, we have analyzed the social, economic and political implications that come with the organization of the production and the appropriation of knowledge in the historically relevant area of agriculture.

Keywords: seeds; science; rice; production; appropriation; technical-scientific knowledge; common goods

Submitted: April 27th, 2017 / Approved: April 26th, 2018

\section{Introducción}

¿Qué implicaciones conlleva la producción de ciencia y tecnología en países que no integran la reducida lista de potencias mundiales? ¿Existe una ciencia hegemónica y una periférica? ¿Qué vinculaciones se establecen entre las comunidades científicas, el mercado, los decisores políticos y las poblaciones implicadas? Estos y otros interrogantes han sido abordados, desde diversos enfoques, tanto dentro del campo de estudios centrado en el análisis de las relaciones entre ciencia, tecnología y sociedad (CTS), como desde distintos aportes disciplinares, provenientes de la economía del cambio tecnológico, la historia social de la ciencia, y las relaciones internacionales, entre otras. Enfocando experiencias latinoamericanas, ha sido discutida la excelencia académica en la periferia en perspectiva histórica (Cueto, 1989), la existencia de "ventanas de oportunidad" (Pérez, 2001), la industrialización de conocimiento en el "centro" (Kreimer, 2011), entre otras temáticas relevantes.

Por otro lado, el extractivismo en América Latina, entendido como una forma específica de explotación y apropiación de la naturaleza, ha sido objeto de numerosos estudios que han indagado sus implicaciones económicas, políticas y culturales. La atención recibida por esta problemática no es casual. Por su disponibilidad de recursos,
América Latina fue, y continúa siendo, una tierra de oportunidades para la rentabilidad capitalista. Según un informe del BID, hasta el 2003 concentraba el $23 \%$ de la tierra cultivable, el $46 \%$ de los bosques naturales y el 31\% del agua dulce del mundo (BID, 2003, p. 19, citado en Manzanal, 2012). Dentro de los trabajos que han tomado al extractivismo como objeto de estudio, se ha analizado en forma creciente la apropiación de conocimientos asociados a la explotación de aguas, tierras, semillas, minerales. En estos trabajos, los conocimientos nativos, originados por comunidades indígenas y campesinas, han recibido una gran atención, ligada a la histórica expropiación de estos saberes (Caldas, 2004; Ribeiro, 2001, entre otros). Por el contrario, la apropiación de conocimientos científicos vinculados a la naturaleza y generados con fondos públicos en los institutos de investigación de América Latina ha sido poco explorada. Sin embargo, también su dinámica de producción se encuentra directamente asociada a conflictos provocados por las tensiones entre las matrices productivas vigentes, y los modos diferenciados de acceso a los bienes comunes naturales. Partiendo de estas problemáticas, este artículo presenta un estudio de caso, a partir del cual se analiza la producción, uso y apropiación de conocimientos científicos obtenidos en Argentina, orientados a la obtención de semillas genéticamente modificadas.

(1) Consejo Nacional de Investigaciones Científicas y Técnicas (CONICET), Centro de Estudios de Historia de la Ciencia y la Técnica José Babini, Universidad Nacional San Martín (UNSAM). E-mail: c.gargano@conicet.gov.ar 
¿Por qué considerar las semillas? En ellas se expresan de manera particular las contradicciones entre rentabilidad y derechos. Son la base de la soberanía alimentaria a escala planetaria y, al mismo tiempo, el eje sobre el que se tejen negocios millonarios que incluyen a la producción semillera en sí, insumos agrícolas ligados vertical y horizontalmente a ésta, y cadenas de producción de conocimientos científicos y tecnológicos que dan como resultado cultivos modificados. Estas tensiones se expresan desde la propia definición de los recursos fitogenéticos. Así, según la Food and Agriculture Organization (FAO), de Naciones Unidas, se denomina "recursos fitogenéticos" a "todo material genético de origen vegetal de valor real o potencial para la alimentación y la agricultura" (FAO, 1996: 3). También remarcando su importancia en la alimentación, el Instituto Nacional de Tecnología Agropecuario (INTA) argentino señala que "Los recursos fitogenéticos son mucho más que insumos básicos para los programas de mejoramiento de las especies cultivadas: son base de la alimentación de la humanidad" (INTA, 2009, p. 2, subrayado propio). Este artículo aborda estas tensiones a la luz de una dinámica de producción científica y tecnológica radicada en el INTA de Argentina. A partir de la reconstrucción de una trayectoria de investigación orientada a la obtención de variedades de arroz resistentes a herbicidas, iniciada en 1996, se analizan conflictos presentes en la utilización comercial de conocimientos científicos generados con fondos públicos. También se señalan vinculaciones entre la forma de organización de la producción del conocimiento, y el modelo extractivista vigente en el agro, en particular en lo referente a la exportación de commodities agrícolas. Para ello, se revisan convenios de vinculación entre el INTA y empresas involucradas en la producción de agroquímicos y semillas, y se discute para qué y para quiénes se orientan los programas de investigación y extensión rural.

En esta dinámica, el Estado es analizado como actor que participa en las controversias, tanto como ámbito en el que se dirimen los conflictos. Por otro lado, el conocimiento científico es entendido como bien común (Coriat, 2015). La noción de "bienes comunes" es una definición de carácter teórico-político, que aboga por una relación no mercantilista y comunitaria de aquellos recursos básicos para la supervivencia y reproducción social de las comunidades (Helfrich y Haas, 2008). La denominación alude metafóricamente a las commond lands, tierras de uso común existentes durante el medioevo europeo en las que existían dinámicas comunitarias de producción y un heterogéneo esquema de relaciones de solidaridad y ayuda mutua. Mientras que la transición del feudalismo al capitalismo tuvo en el proceso de cercamiento de tierras un hito fundamental, la literatura que aborda el estudio de los comunes ha analizado diversos procesos de cercamientos que avanzan en la privatización de elementos fundamentales para el desarrollo de la vida humana. Dentro de este enfoque, en el que cabe mencionar los aportes pioneros de Ostrom (2000), una gran cantidad de trabajos analizan la producción de conocimiento y los sistemas de "acceso abierto", desde el punto de vista de la producción académica, la generación de datos informáticos, y de diversos procesos productivos enmarcados en la era del denominado "capitalismo cognitivo" (Vercellone, 2011). ${ }^{1}$ Por otro lado, una serie de estudios analizan la apropiación de los bienes comunes naturales, como el agua, la tierra, los minerales, la energía y las semillas. En este artículo abordamos una transición entre ambos ejes analizando una producción cognitiva asociada a un bien común natural, como entendemos son las semillas.

El primer apartado presenta el caso de estudio, describiendo las principales características de la trayectoria de investigación estudiada. El segundo, analiza la apropiación y comercialización de los resultados, problematizando la mercantilización del conocimiento. El tercero presenta un breve recorrido por la historia de la tecnología agropecuaria en América Latina, señalando sus implicancias políticas, económicas y culturales. En las conclusiones se exponen los resultados obtenidos. A partir de materiales documentales y de entrevistas a técnicos y científicos, se analiza así una dinámica de investigación centrada en el cultivo comestible más importante del mundo, el arroz.

\section{Trayectoria de una investigación}

El arroz es una planta herbácea anual, de la misma familia que el trigo, la avena o el centeno. En términos económicos y sociales, posee una importancia singular: es el cultivo comercial comestible más importante del mundo. Es decir, forma parte de uno de los principales engranajes de producción y comercialización de mercancías primarias, de donde derivan ganancias millonarias que son concentradas por un puñado de empresas transnacionales. Y, al mismo tiempo, es una pieza fundamental para la soberanía alimentaria. La investigación orientada al mejoramiento genético en este cultivo posee una larga trayectoria en América Latina. En particular, dentro del INTA argentino, el primer organismo abocado a la investigación y extensión rural de toda la región, diversos equipos de técnicos se dedicaron a su estudio y experimentación. Dentro de las trayectorias de investigación recientes, uno de ellos logró alcanzar un resultado único a nivel mundial: la obtención de variedades de arroz resistentes a herbicidas del grupo de las imidazolinas. ¿Qué características tuvo este desarrollo? ¿Y qué impacto supuso a nivel internacional?

Existen tres tipos de tecnologías utilizadas para inducir la resistencia a herbicidas en arroz. Una de ellas se orienta a la resistencia al glufosinato, el herbicida es comercializado bajo la marca Liberty Link ${ }^{\star}$ por la empresa multinacional Bayer CropScience. La segunda induce resistencia al herbicida glifosato, que es vendido con la marca comercial Roundup Ready ${ }^{\circledast}$ por la firma Monsanto. La reciente fusión entre ambas firmas, liderada por Bayer, reduce aún más este esquema. Finalmente, la tercera genera resistencia a herbicidas de la familia de las imidazolinonas, es conocido comercialmente como Clearfield ${ }^{\circ}$ ("campo limpio") y comercializado por la empresa BASF. Ésta última opción fue la elegida por el INTA.

Los investigadores buscaron obtener variedades de arroz con este tipo de resistencia, ya que la misma es obtenida por mutagénesis, un

(1) Para una revisión crítica de diversas perspectivas ligada al capitalismo cognitivo, véase la realizada por Sztulwark y Míguez (2012). 
proceso que no implica la implantación de un gen extraño a la planta. A diferencia de lo sucedido en las otras dos opciones, en este caso la nueva semilla obtenida no es considerada por la regulación vigente un organismo genéticamente modificado (OGM), ya que la planta resultante no recibe un gen de resistencia al herbicida que proviene de otro organismo. Esta característica, buscada por la restringida aceptación de los transgénicos a nivel mundial, fue una de las razones de peso, sumada también a su factibilidad y propiedades. En palabras del director del equipo de trabajo, el Dr. Alberto Livore, "Elegimos esos herbicidas por sus propiedades, tanto como controladores de maleza como también por el bajo impacto ambiental, y porque existían antecedentes en otras especies que nos hacían pensar que era posible" (Livore, 2013, entrevista). Así, la efectividad en el control de un amplio espectro de malezas, como el arroz colorado, cyperaceas y gramíneas perennes, sumado a un alto rendimiento y al hecho de implicar la obtención de un cultivo transgénico, aparecen como los principales argumentos en la elección.

Radicado en la estación experimental agronómica de Concepción del Uruguay del INTA, situada en la provincia de Entre Ríos, en el noreste argentino, el equipo de investigación comenzó las investigaciones en el año 1996. En el equipo convivieron perfiles profesionales distintos, que garantizaron un tratamiento integral de las nuevas variedades. De sus doce integrantes, solamente cuatro se dedicaron a fitomejoramiento, los demás se especializan en ecofisiología y patología vegetal, como estrategia para garantizar que las variedades tengan todos los atributos propios de un alto rendimiento. En cuanto al proceso de mejoramiento, la utilización de herramientas biotecnológicas, como los marcadores moleculares, fueron un elemento estratégico para la reducción de tiempos, por posibilitar la identificación temprana de características de difícil detección. Sin embargo, según los investigadores, el núcleo duro del trabajo sigue siendo la selección tradicional. En otras palabras, el ojo del mejorador no ha sido, al menos todavía, suplantado por otros procedimientos.

Luego de más de una década de pesquisas, el equipo obtuvo cuatro mutantes, de los cuales lograron patentar dos. "Puitá INTA CL", en el 2004, es una variedad de arroz de ciclo corto, alto rendimiento agrícola y calidad culinaria. Fue inscripta en el Instituto Nacional de Semillas (INASE) el 22 de agosto de 2005. "Gurí-INTA CL", la segunda variedad, fue inscripta en el INASE en febrero del 2011. Esta último mejoró el rendimiento de su antecesora, combinando las características de resistencia a herbicidas con las de alta calidad industrial y culinaria de la variedad. Ambas fueron las primeras variedades de alto rendimiento y calidad, con adaptación a climas subtropicales y tropicales, resistentes al herbicida conocido comercialmente como "Clearfield". Como señalamos, el hecho de tratarse de variedades no transgénicas permite su comercialización sin trabas hacia los países que levantan barreras contra estos cultivos.

A nivel mundial, existía un único desarrollo similar al alcanzado por los investigadores del INTA. También producido en una institución oficial, en este caso la Universidad de Louisiana, en Estados Unidos. Dos años antes que se conocieran los resultados del INTA, esta Universidad comunicaba que habían logrado un mutante resistente. Sin embargo, el equipo argentino logro obtener la patente ya que pudo probar que obtuvo un mutante distinto. Según explica el director del equipo, "Nosotros probamos que nuestro mutante era diferente porque la mutación se encuentra en otro lugar del gen y de esa manera se pudo obtener la patente" (Livore, entrevista, 2013).

Culminada la investigación, con el consecuente prestigio y reconocimiento académico conseguido por los investigadores argentinos, restaba un paso fundamental. ¿Qué ocurriría con los resultados de la investigación que finalmente, luego de años de sostenidas inversiones, habían sido alcanzados?

\section{Mercantilización de la ciencia y privatización de las semillas}

A lo largo de la investigación orientada a desarrollar variedades de arroz resistentes a un grupo de herbicidas, el INTA contó con dos socios del sector privado. El primero, la Fundación ProArroz, una organización creada en 1994 por importantes capitales de la cadena del arroz (semilleros, molinos, multiplicadores), cooperativas de productores y el propio INTA, con el objetivo de "promover el desarrollo del cultivo del arroz a través de la generación y difusión de tecnologías", según su propio estatuto. El aporte de recursos financieros por parte de la Fundación es destacado por los integrantes del Programa de Mejoramiento de arroz de INTA, porque "cubrieron los altibajos que normalmente se producen en las instituciones públicas" (Livore, entrevista, 2013). En octubre de 2004, el organismo y la Fundación firmaron un convenio para la producción y multiplicación de las variedades liberadas. Así, en Argentina, la producción de semilla original de estas variedades está a cargo de esta Fundación, al igual que el cobro de regalías. Es la única productora de semilla original autorizada para la venta de las variedades de INTA a los semilleros fiscalizados inscriptos en el INASE. Mientras que a nivel local la multiplicación y venta es responsabilidad de la Fundación, un tercer participante para su expansión comercial internacional es la empresa BASF.

De origen alemán, BASF es una multinacional que lidera la industria química a nivel mundial. Creada en 1865, posee plantas productivas y oficinas de venta a lo largo del mundo. Es, además, propietaria de los herbicidas que fueron utilizados por los investigadores del INTA como objetivo para obtener la resistencia. El interés de la firma en estas investigaciones era directo: al momento de disponer de un arroz resistente, aumentaría el mercado de sus herbicidas. Las variedades desarrolladas por el INTA son resistentes al herbicida Kifix ${ }^{\oplus}$, desarrollado y comercializado por BASF.

Mientras que en un inicio la firma participó en la tarea de secuenciar los genes obtenidos, lo que permitió identificar qué mutaciones eran y su originalidad, una vez obtenidos los resultados, avanzó en un acuerdo comercial. El INTA le otorgó a la empresa la exclusividad de la licencia de uso del gen en todo el mundo, exceptuando a la Argentina y Uruguay, donde retuvo la administración de los derechos de propiedad de la patente. El Convenio firmado entre ambos, posee 10 años de duración, con opción a renovación. De esta forma, la difusión y comercialización a nivel mundial corre por cuenta de una empresa transnacional. 


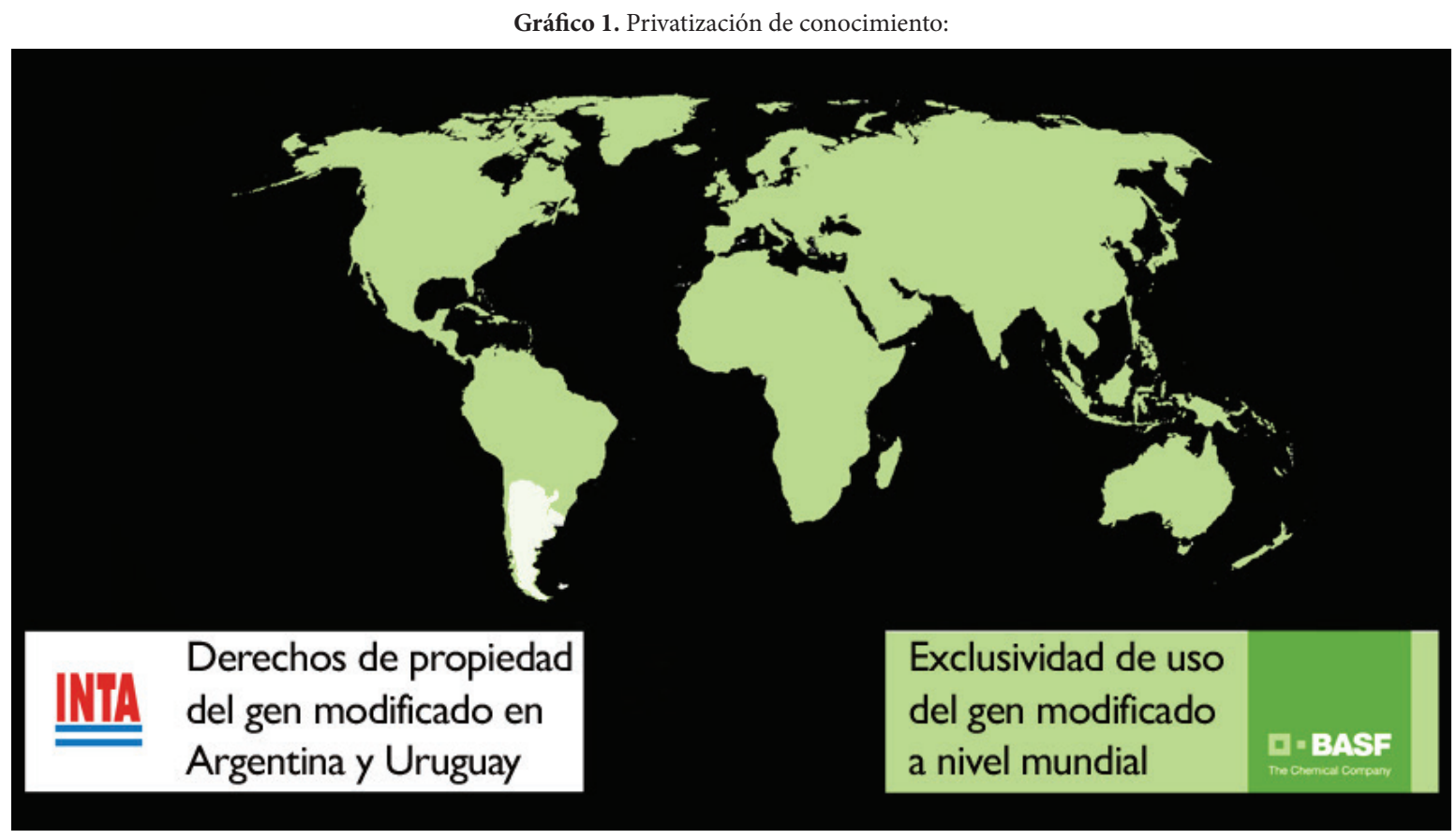

Fuente: Gárgano (2013)

Los resultados de la investigación fueron recibidos como un verdadero éxito. Tanto para los investigadores en particular, como para el INTA y los funcionarios del sector agropecuario en general, éste radicaba en su proyección internacional. Así, que las variedades pudieran ser comercializadas en todos los países productores de arroz, demostraba la capacidad de generación de tecnología de impacto por parte del INTA. Esta visión también está presente en la literatura académica. En este sentido, el trabajo de Fuck y Machado Bonacelli (2009), quienes realizaron un estudio comparativo entre el INTA y Embrapa, tomó a este mismo convenio entre INTA y BASF como un ejemplo de articulación público-privada virtuosa. Si bien no analizaron la trayectoria de la investigación y se limitaron a mencionar la firma del convenio, destacaron que tanto éste como otros acuerdos similares le permitirían al INTA "expandir sus actividades de investigación", y tener "una posición de liderazgo internacional" (Fuck \& Machado Bonacelli, 2009, p. 39). Así, en la tesis de los autores todas las partes saldrían beneficiadas: los "socios" (las empresas) y los productores (quienes con mayores rendimientos alcanzarían mejores ganancias), y el INTA. Cabe preguntarnos, ¿es real esta imagen sin tensiones aparentes? ¿Todos ganan? La caracterización de los académicos no solamente reproduce los argumentos de los propios científicos, también omite especificar qué tipo de productores son los que pueden acceder al paquete tecnológico y alcanzar mejores rendimientos. Y, fundamentalmente, deja en un mismo plano a los beneficios alcanzados por la firma transnacional, BASF, y a los recibidos por el organismo de investigación local en concepto de regalías. Sin embargo, la relación dista de ser simétrica.

En el 2014, BASF obtuvo ganancias por 74 millones de euros. Solamente la división "Productos Agrícolas" generó ventas por 3.354 millones. Según su propio portal, la firma tiene como objetivo "convertir rápidamente el conocimiento en éxito de mercado". Ciertamente, éste parece haberse cumplido en el acuerdo alcanzado con el INTA. Al mismo tiempo, afirman que su liderazgo en innovación agrícola permite optimizar la producción "mejorando la nutrición y por lo tanto, realzando la calidad de vida para la creciente población mundial”. En esta línea, afirman que a través de la ciencia y la innovación, ayudamos a nuestros clientes de casi todas las industrias a satisfacer las necesidades presentes y futuras de la sociedad" (BASF, web). Sobre este punto, la evidencia no abunda.

\section{Tecnología agrícola en América Latina: ¿saberes colonizados?}

Durante milenios la domesticación de plantas fue realizada en forma colectiva, generando un progresivo incremento de los rendimientos de la mano de múltiples procesos de cruza y selección. Desde los comienzos de la sedentarización y la agricultura hasta nuestros días, hace más de 10.000 años, la humanidad ha generado y acumulado un caudal inmensurable de conocimiento. Éste ha atravesado profundas transformaciones, en consonancia con los cambios que, a lo largo de la historia, se han producido en las relaciones sociales. Dentro de estas grandes etapas históricas, la aparición de la ingeniería genética, en el siglo XX, marcó un antes y un después en la relación entre el hombre y la naturaleza, así como en los entramados entre ciencia y cultura, y en las relaciones entre ciencia y mercado (Palladino, 2002).

Entre 1943 y1961, un programa de investigación agrícola y asistencia técnica desarrollado por los Estados Unidos fue puesto en marcha en México. Con él se originó el paquete tecnológico de la llamada revolución verde, que sería exportado a América Latina, Asia e India, con un profundo impacto tanto en los rendimientos de algunos de los principales cultivos, como en las distintas estructuras sociales 
agrarias. El término "Revolución Verde" fue utilizado por primera vez en 1968 por el ex director de la United States Agency for International Development (USAID), William Gaud, quien destacó que la difusión de las nuevas tecnologías "y otros desarrollos en el campo de la agricultura contienen los ingredientes de una nueva revolución. No es una violenta revolución roja como la de los soviéticos, ni es una revolución blanca como la del Sha de Irán. Yo la llamo la revolución verde." Estudios como el de Fitzgerald (1986) han señalado que los programas iniciales fueron exitosos en las regiones mexicanas en las que las condiciones ambientales y socio-económicas eran similares a las del agro estadounidense. Así, mientras que la obtención y difusión de nuevos trigos fue exitosa, no ocurrió lo mismo con los maíces híbridos, compatibles con los farmers y con un agro capital intensivo pero no con la agricultura de subsistencia del campesinado mexicano (Fitzgerald, 1986). El modelo de investigación y extensión rural propagado desde el Centro Internacional de Mejoramiento en Maíz y Trigo (CIMMYT) tuvo una gran impronta en la agricultura a nivel mundial. Su difusión implicó una transformación radical de la agricultura, dominada por la mecanización y la generación de semillas genéticamente modificadas de alto rendimiento, unidas a un paquete de fertilizantes, pesticidas y herbicidas. Estos cambios estuvieron acompañados por la expansión a nivel mundial de la industria química, bioquímica y farmacológica. Diversos autores han analizado críticamente el fenómeno. Entre los puntos señalados, se ha hecho hincapié en que con el argumento de contrarrestar las hambrunas en distintas regiones, la modernización tecnológica del agro tuvo como contrapartida la aparición de fuertes transformaciones sociales, económicas y ambientales (Pengue, 2005). También se ha señalado que fue convergente a la movilidad de capitales -en ascenso luego de la caída de Bretton Woods- que fomentó el accionar de grandes firmas, empresas trasnacionales, laboratorios químicos, semilleras y comercializadoras exportadoras, que pasaron a controlar la mayor parte del mercado agrícola internacional (Teubal, 2001). En este escenario, el uso de insumos externos que conformó el paquete tecnológico (agroquímicos, semillas mejoradas y renovadas técnicas de irrigación) se incrementó notablemente (Perelmuter, 2011). En Argentina, el INTA fue un activo introductor de los "trigos mexicanos", que fueron cruzados con variedades locales, y en la producción propia de híbridos de maíz.

A nivel internacional, distintos analistas de la ciencia ubican en la década de 1970 el avance hacia la privatización de la ciencia y la tecnología (Pestre, 2005). Para la década de 1980, en Estados Unidos, dos relevantes transformaciones del marco regulatorio de la actividad de CyT se vincularon a esta problemática. El Acta de Transferencia de Tecnología de Stevenson-Wydler, que facilitó los convenios entre laboratorios públicos, universidades y empresas, y la Enmienda Bayh-Dole a las leyes de patentes, que otorgó a las universidades y centros de investigación la posibilidad de percibir derechos de propiedad intelectual por trabajos realizados con fondos públicos (Krimsky, 1991). Durante este período, estas tendencias internacionales en la producción científica en Argentina se combinaron con un modelo económico aperturista, y con una fuerte concentración de capital en el agro protagonizada por empresas transnacionales semilleras y agroquímicas. La escala tecnológica y económica requerida para los nuevos paquetes tecnológicos (semillas + agroquímicos) relegó al país al rol de receptores de esas tecnologías (Bisang y Varela, 2006). Sin embargo, en algunos nichos de investigación agrícola, como lo es el caso estudiado en este artículo, el Estado mantendría posiciones de liderazgo internacional. Mientras que, a lo largo de la década de 1960, las investigaciones oficiales apuntalaron la consolidación de la industria semillera local (sin ningún tipo de retorno por la utilización de las investigaciones), en el nuevo escenario los conocimientos generados se articularon a un escenario transnacional. El fin de los '80, en plena crisis inflacionaria, vio nacer los "Convenios de Vinculación Tecnológica” (CVT) con empresas. El pionero fue el INTA. En este sentido, la articulación analizada, entre la empresa BASF y el INTA, fue realizada utilizando esta figura de la política de vinculación tecnológica vigente, que el organismo inauguró oficialmente en 1987. Mediante estos convenios estableció acuerdos con el sector privado, que a cambio de financiar parte de las investigaciones, accedió en forma explícita a la utilización comercial de los resultados. Estos "desarrollos conjuntos" le sirvieron al INTA para garantizar su supervivencia durante el brutal recorte al presupuesto registrado en la década de 1990, y formalizaron una relación que, en los hechos, ya estaba desplegándose.

En un estudio previo, centrado en la producción de híbridos de maíz en Argentina, indicamos la existencia de un ciclo histórico de apropiación privada de conocimientos generados con fondos públicos, por parte de un sector concentrado del sector semillero, y señalamos la existencia de tres hitos relevantes en el marco regulatorio (Gárgano, 2016). El primero, durante la década de 1960, en el momento de conformación de la industria semillera local, cuando una disposición de la Secretaría y Agricultura estableció en 1959 el secreto comercial de los híbridos del sector privado (el pedigree cerrado), mientras que obligó a que los híbridos del sector público mantuvieran disponible la información de sus fórmulas (pedigree abierto), sin percepción de regalías, generando que los híbridos de la naciente industria semillera fueran registrados como propios a partir de las investigaciones realizadas en el sector oficial. El segundo, en 1979, cuando en tiempos de la intervención del INTA en el marco de la última dictadura militar (1976-1983), una resolución del organismo dictaminó la cesión de sus recursos fitogenéticos a los criaderos privados. Y, el tercero, poco antes de iniciar la década de 1990, con la implementación de los CVT. El presente estudio, centrado en una investigación reciente, permite identificar la continuidad y vigencia de esta tendencia de direccionamiento de los resultados de las investigaciones en semillas realizadas con fondos públicos.

Cabe precisar que dentro de la producción científica y tecnológica estas tendencias no son privativas del INTA. Al contrario, se condicen con las pautas explícitas e implícitas de buena parte de las instituciones y marcos regulatorios locales. Al mismo tiempo, el protagonismo de firmas como BASF en los convenios de vinculación tecnológica, tampoco es una singularidad de la investigación local, ni de los patrones de producción vigentes en el espacio rural argentino. El crecimiento del agronegocio ha coincidido con una fuerte concentración, de la que 
BASF es parte junto a un selecto grupo de firmas. Para el año 2015, solamente tres empresas (Monsanto, Dupont y Syngenta) controlaban el $55 \%$ del mercado mundial de semillas, mientras que seis (Bayer, Monsanto, Dupont, Dow, Basf y Syngenta), dominaban el 75\% del mercado de agroquímicos (Ribeiro, 2001). A este panorama se le ha añadido la fusión, producida en septiembre de 2016, entre Bayer y Monsanto, protagonizada por la primera. El 65,4\% de la producción de agroquímicos permanece actualmente concentrada en tres empresas, ChemChinaSyngenta (25,8 \%), Bayer-Monsanto (24,6\%) y DuPont-Dow (15\%). En el área de semillas, el 60,7 \% del mercado queda a cargo de las mismas firmas: Bayer-Monsanto (30,1\%), DuPont-Dow $(22,7)$ y ChemChinaSyngenta (7,9\%). En este marco de progresiva concentración horizontal y vertical, se multiplica la utilización de germoplasma proveniente del sur. En simultáneo, las nuevas leyes de semillas impulsadas en distintos países de la región amenazan con poner fin a las exenciones a los agricultores, el histórico "uso propio", y a los investigadores, los intercambios no lucrativos con fines de investigación, ambas contempladas en las legislaciones vigentes. En este proceso, el derecho de "participar en el progreso científico y en los beneficios que de él se derivan", in corporado en las declaraciones universales de DDHH (Ribeiro, 2001, p. 363), está tan lejos de ser alcanzado como lo está el replanteo de las condiciones de producción en el espacio rural.

Los modelos extractivistas se han articulado en base a un binomio constituido entre los poderes financieros transnacionales y los gobiernos locales, que ha dado como resultado un patrón concentrador y excluyente, con altos costos sociales y ambientales (Manzanal, 2012). En este sentido, es posible señalar una articulación entre la acumulación por desposesión que describe David Harvey (2004) y el "consenso de las commodities" señalado por Maristella Svampa (2013), en la que, junto a la explotación de la naturaleza, la dimensión cognitiva juega un rol singular. En articulación con estas dinámicas, se evidencia la transformación de bienes comunes naturales en commodities agrícolas y también el carácter conocimiento intensivo de estas mercancías agrarias. Así, esta acumulación por desposesión incluye también a la producción local de conocimiento. Y, en rigor, en el plano analizado, cabe revisar la categoría de "desposesión", ya que lo encontramos en una cesión, legalmente regulada e impulsada por el Estado.

\section{Conclusiones ( o ciencia para qué y para quiénes)}

Actualmente, las tres tecnologías dirigidas a lograr variedades de arroz resistentes a herbicidas involucran a grandes empresas transnacionales. El INTA argentino, en un desarrollo pionero, obtuvo nuevas variedades que se están difundiendo en América, Asia y Europa. Argentina es también el octavo productor mundial de arroz, el cultivo comestible más importante del mundo. Como se ha reconstruido, el proceso de investigación y obtención de estos resultados involucra a una de las principales firmas transnacionales del concentrado mercado semillero y agroquímico, BASF. El caso estudiado expone las tensiones existentes entre la conceptualización de las semillas como bienes comunes, pilares en la reproducción de la humanidad, y su transformación en mercancías. Y muestra cómo, en este proceso histórico, la mercantilización del conocimiento científico asociado a la obtención de nuevas variedades posee relevantes derivaciones.
Sheldon Krimsky (1991) ha caracterizado como "capitalismo académico" a los patrones de producción de conocimiento que proliferaron como consecuencia de la imbricación creciente entre universidades y empresas. En rigor, la definición excede al ámbito universitario. En Argentina, esta problemática es indivisible de su matriz de productiva, por lo que los conocimientos vinculados a la producción agrícola juegan un rol primordial. En el convenio de vinculación analizado entre el INTA y la firma BASF, el beneficio institucional redunda en el reconocimiento internacional de la genética de arroz desarrollada por el organismo, y en la obtención de regalías, (magras en comparación con las ganancias millonarias derivadas de la comercialización de las variedades a nivel mundial), para seguir trabajando en el programa de mejoramiento. En este sentido, cabe preguntarnos, ¿es ésta la única posibilidad de direccionamiento (y por lo tanto de apropiación) del conocimiento científico y tecnológico generado en el ámbito público? Junto al prestigio internacional que logran de este modo las investigaciones, ¿no sería deseable retener en la misma esfera pública que ha sostenido por años las investigaciones y proporcionado la materia prima (el conocimiento) para la obtención de las variedades comerciales? No casualmente los dos centros a la vanguardia a nivel mundial en esta área son organismos estatales, de donde proviene el grueso de las inversiones. Aun considerando que en Argentina los períodos de ajuste presupuestario impulsaron que la continuidad de los programas descansara en los aportes de socios privados, sería necesario no solamente que ésta estuviera garantizada desde el sector público, sino que además, y en forma primordial, las prioridades de las agendas de investigación respondiesen a las necesidades de las poblaciones involucradas. ¿Cuánta utilidad les reportan a las comunidades rurales los convenios de vinculación con firmas transnacionales, que generan semillas genéticamente modificadas para ser resistentes a los herbicidas que estas mismas empresas producen? Esta ecuación resulta inviable mientras las propias comunidades no sean incorporadas al esquema, binario y al mismo tiempo en permanente retroalimentación, falazmente denominado "público-privado". Al mismo tiempo, ¿en qué medida el Estado argentino es capaz de recaudar fondos derivados de las investigaciones agrícolas que promueve para incorporarlos al gasto público en general y a los agricultores en particular? Tampoco esta pregunta tiene una respuesta posible mientras la agenda continúe definida en función de los requerimientos de las grandes corporaciones.

La ausencia histórica de políticas agropecuarias que promuevan emprendimientos para otorgar una continuidad al conocimiento producido en el ámbito público, a través por ejemplo de un organismo como el INTA, aparece también como un desafío pendiente. Y aún más, el modo en el que se definen los contenidos y las prioridades de las investigaciones. En este sentido, si el Estado, además de garante de un orden social, es considerado como una arena en disputa (De Sousa Santos, 2006), el ámbito de producción científica no debería ser dejado fuera de la agenda de preocupaciones a la hora de discutir una agricultura alternativa a los actuales modelos de explotación del suelo y expulsión de los pobladores del campo. Por un lado, por los patrones culturales, económicos y políticos que han atravesado los programas de investigación en fitomejoramiento impulsados en América Latina desde la revolución verde. Por otro, porque el direccionamiento y construcción de las agendas de investigación y extensión rural 
es también un terreno en disputa. Al fin y al cabo, la ciencia es una construcción colectiva y tanto el proceso social de creación de conocimiento como su potencialidad se rompen cuando se subordinan a la lógica sustentada en la búsqueda de ganancia, que por definición se opone a la resolución de necesidades sociales. Repensar las formas de producción y de vida en el ámbito rural también requiere revertir la tendencia hacia apropiación privada del conocimiento orientado a la producción agrícola que es generado con fondos públicos.

Analizar críticamente la construcción de los contenidos y beneficiarios de los programas de investigación en semillas vigentes en Argentina implica, por un lado, reflexionar en torno a las posibles implicancias de estas problemáticas en la construcción de un modelo agrícola alternativo, que priorice la concepción de las semillas, y de los múltiples saberes asociados a ellas, como bienes comunes. Por otro, expone las implicaciones vigentes en la producción científica contemporánea. En este sentido, lejos de dirimirse en una polarización binaria entre una "ciencia hegemónica" -producida en las principales potencias mundiales- y una "ciencia periférica"- supeditada a esta primera y realizada en el denominado "tercer mundo" o bien en el heterogéneo conjunto englobado como "países en desarrollo"- encontramos una compleja trama que vincula ambos espacios en forma asimétrica pero promoviendo una misma ciencia empresarial, alejada de las necesidades de los territorios. En este proceso, las alianzas entre comunidades de investigación y empresas transnacionales exponen que es el Estado quien financia las rentas privadas (Dagnino, 2009). Resta analizar, en este sentido, los procesos de validación de los conocimientos generados incorporando tanto las tensiones entre diversas nociones de derechos (humanos, ambientales y de propiedad intelectual), como las derivadas de las presiones de la economía global. También se encuentra pendiente el análisis dentro la producción científico-tecnológica estudiada de los riesgos potenciales que implican al ambiente y a las poblaciones, y del rol de científicos y organismos estatales en su omisión.

Finalmente, este estudio de caso permite afirmar que la dimensión cognitiva es parte constitutiva de los modelos extractivistas y de las relaciones neocoloniales que los constituyen. La actual forma de organización de la producción de conocimiento científico y tecnológico en América Latina se encuentra atravesada por la configuración de sus matrices productivas. En este sentido, la intensificación del proceso de despojo y acumulación de capital a nivel mundial nos reserva a los países "periféricos" una tarea clara, histórica y renovada. Detrás de la llamada a que estos países apuntalen sus "sistemas nacionales de innovación" reaparece el mismo rol: la extracción creciente de bienes comunes naturales, acompañada cada vez más por la fuga de resultados de investigación. Más específicamente, de las ganancias derivadas de estos resultados. La semilla, patrimonio de la humanidad desde hace miles de años, no fue la única que se transformó en mercancía. Más lentamente, también lo hizo el propio conocimiento científicotecnológico. En la Argentina del agronegocio, la ciencia empresarial juega un rol fundamental que necesitaremos revisar si queremos transformar las profundas asimetrías vigentes y construir nuevos modos de habitar, producir y vivir.

\section{Acerca de la autora}

Cecilia Gárgano es Doctora en Historia por la Universidad de Buenos Aires (UBA). Realizó su investigación doctoral y postdoctoral como becaria del Consejo Nacional de Investigaciones Científicas y Técnicas (CONICET), donde fue seleccionada como investigadora de la Carrera de Investigación Científica (CIC) en 2016. Profesora de la Escuela de Humanidades e investigadora del Centro de Estudios de Historia de la Ciencia y la Técnica "José Babini” (UNSAM). Integra el Grupo de Trabajo "Bienes Comunes" del Consejo Latinoamericano en Ciencias Sociales (CLACSO). Se dedica al análisis de la producción y apropiación de conocimiento científico orientado al agro, y a la historia reciente de la ciencia en Argentina. Desde el 2014 dirige un Proyecto INTA-CONICET, surgido de su tesis doctoral, y desde el 2016, el Proyecto PICT “Ciencia y Política en Argentina: producción pública de semillas y apropiación privada de conocimiento" financiado por la Agencia Nacional de Promoción Científica y tecnológica (ANPCyT).

\section{Bibliografía}

BASF (s/a) "Acerca de BASF en el mundo", sitio web http://www.basf. com.ar/sac/web/argentina/es_ES/acerca-de-basf/basf-en-el-mundo, consultado el 10-03-2017

Bisang, Roberto y Varela, Liliana (2006). "Panorama internacional de la biotecnología en el sector agrario. Dinámica de las mega empresas internacionales de agrobiotecnología e impacto sobre la oferta local". En: Bisang, R., Gutman, G. E., Lavarello, P., Sztulwark, S. y Díaz, A. (comps.) Biotecnología y desarrollo. Un modelo para armar en la Argentina, pp.25-62. Buenos Aires: Prometeo.

Caldas, A. (2004). La regulación jurídica del conocimiento tradicional: la. La Conquista de los Saberes. Bogotá: ILSA.

Coriat, Benjamin (2015). Le retour des communs. La crise de l'idéologie propriétaire, Paris: Éditions Les Liens qui libèrent.

Cueto, Marcos (1989). Excelencia científica en la periferia: actividades científicas e investigación biomédica en el Perú 1890-1950. Lima: Grade-CONCyTEC.

Dagnino, Renato (2009). "A construção do Espaço Ibero-americano do Conhecimento, os estudos sobre ciência, tecnologia e sociedade e a política científica e tecnológica”, Revista CTS, (4) 12, pp. 93-114.

De Sousa Santos, Boaventura. (2006). Reinventar la democracia. Reinventar el Estado. Buenos Aires: CLACSO.

FAO - Dirección de Producción y Sanidad Vegetal (1996, junio). "Informe sobre el estado de los recursos fitogenéticos en el mundo", Conferencia Técnica Internacional sobre los Recursos Fitogenéticos. Leipzig: FAO.

Fitzgerald, D. (1986). Exporting American Agriculture: The Rockefeller Foundation in Mexico, 1943-53. Social Studies of Science, 16(3), 457-483. doi:10.1177/030631286016003003 
Gárgano, Cecilia (2013). "Aportes del sector público a la producción de arroz" Tecnología Sur-Sur, disponible en: http://www.unsam.edu. ar/tss/aportes-del-sector-publico-a-la-produccion-de-arroz/

Gárgano, Cecilia (2016). “Genética vegetal: conformación de un campo de estudios y de una trayectoria histórica de apropiación de conocimiento", en Kreimer, P. (comp.) Contra viento y marea. Emergencia y desarrollo de campos científicos en la periferia, Buenos Aires: CLACSO, pp. 149-178.

Harvey, David (2003). The new imperialism. Oxford University Press: New York.

Helfrich, Susan y Hass, J. (2008) "Genes, bytes y emisiones: acerca del significado estratégico del debate de los bienes comunes”, en. Helfrich, S. (comp.). Genes, bytes y emisiones: bienes comunes y ciudadanía. México: Ediciones Boll, pp. 311-328.

Instituto Nacional de Tecnología Agropecuaria (2009). Banco activo Pergamino. Pergamino: Ediciones INTA.

Kreimer, P. (2011). Internacionalização e tensões da ciência latinoamericana. Ciência e Cultura, 63(2), 56-59. doi:10.21800/s000967252011000200018

Krimsky, Sheldon (1991). "The profit of scientific discovery and its normative implications", Chicago Kent Law Review, 75 (3), pp. 15-39.

Manzanal, Mabel (2012). "Poder y desarrollo. Dilemas y desafíos frente a un futuro ¿cada vez más desigual?”, en Manzanal, M. y Ponce, M. (org,) La desigualdad ¿del desarrollo? Controversias y disyuntivas del desarrollo rural en el norte argentino. Buenos Ares: Ciccus, pp. 17-49.

Ostrom, Elinor (2000). El gobierno de los bienes comunes. La evolución de las instituciones de acción colectiva. México DF: UNAM-CRIMFCE.

Palladino, Paolo (2002). Plants, Patients and the Historian: (Re)membering in the Age of Genetic Engineering. Manchester: Manchester University Press.
Pengue, Walter (2004) La transnacionalización de la agricultura y la alimentación en América Latina. Informe regional (Buenos Aires: Red de Formación Ambiental para Latina y el Caribe (RFA), Programa de Naciones Unidas para el Medio Ambiente).

Pestre, Dominique (2005) Ciencia, dinero y política. Buenos Aires: Nueva Visión.

Perelmuter, Tamara (2011) "Bienes comunes vs. mercancías: las semillas en disputa. Un análisis sobre del rol de la propiedad intelectual en los actuales procesos de cercamientos", Sociedades Rurales de Producción y Medio Ambiente, 11 (22), 54-86.

Pérez, Carlota (2001). "Cambio tecnológico y oportunidades de desarrollo como blanco móvil”. Seminario sobre la Teoría del Desarrollo en los Albores del Siglo XX, 28-29 agosto 2001, Santiago: CEPAL. Diponible en: http://repositorio.cepal.org/handle/11362/34861

Ribeiro, Silvia (2001). "Propiedad intelectual, recursos genéticos y conocimientos tradicionales". Comercio, Medio Ambiente y Desarrollo Sustentable, pp. 363-380.

Sztulwark, Sebastián y Míguez, Pablo (2012). “Conocimiento y valorización en el nuevo capitalismo”. Realidad económica, 270, pp. 11-32.

Teubal, Miguel (2001). “Globalización y nueva ruralidad en América Latina”, en Una nueva ruralidad en América Latina, pp. 45-65. Buenos Aires: CLACSO.

Svampa, Mariestella. (2013). "Consenso de los Commodities y lenguajes de valoración en América Latina”, Nueva Sociedad, 244, 30-46. Vercellone, Carlo (2011). Capitalismo cognitivo. Renta, saber y valor en la época posfordista. Buenos Aires: Prometeo.

Entrevista al Dr. Livore, INTA, Buenos Aires, 01-08-2013; 04-05-2015.

Entrevista a responsable del banco de germoplasma EEA, Pergamino, Pergamino, 07-06-2016.

Entrevista a integrante del área de Vinculación Tecnológica del INTA, Buenos Aires, 03-05-2016. 
